\title{
Generic Triviality of Phase Diagrams in Spaces of Long-Range Interactions
}

\author{
Robert B. Israel* \\ Department of Mathematics, University of British Columbia, Vancouver, B.C., Canada, V6T 1Y4
}

\begin{abstract}
We show that interactions with multiple translation-invariant equilibrium states form a very "thin" set in spaces of long-range interactions of classical or quantum lattice systems. For example, generic finite-dimensional subspaces do not intersect this set. This constitutes a severe violation of the Gibbs Phase Rule.
\end{abstract}

\section{Introduction}

The work of Daniels and van Enter [1-3] has shown that phase transitions are less stable under long-range perturbations than had been believed, so that Ruelle's "heuristic theory of phase transitions" [7], and the strong form of the Gibbs Phase Rule that it implies, are violated in spaces of long-range interactions. In this paper we extend and generalize those results, and show that the instability of phase transitions is a generic phenomenon, in the sense of Baire category, in these spaces of interactions. This means that phase transitions occur only on a set which is in a sense very "thin," violating much weaker versions of the phase rule.

We will consider either a classical or quantum lattice system on $\mathbb{Z}^{d}$ (see [4] for notation). In the classical case the configuration space at each site is assumed to be a compact metric space. The Banach space $\mathscr{B}_{g}$ consists of those translationinvariant interactions $\Phi$ with

$$
\|\Phi\|_{g} \equiv \sum_{0 \in X} g(|X|)\|\Phi(X)\|<\infty
$$

where $g$ is some function on the positive integers, and $|X|$ is the cardinality of $X$. In order to define equilibrium states by the variational principle, we require $g(n) \geqq 1 / n$ [in the case $g(n)=1 / n$ we will write $\mathscr{B}_{g}$ as $\mathscr{B}$ ]. Stronger conditions on $g$ [e.g. $g(n) \geqq e^{a n}$ for some $a>0$ ] allow the use of DLR equations, KMS conditions, etc. Our results hold in any of these spaces, and thus are not connected to the

* Research supported by NSERC grant A-4015 
pathologies found in [5] for $\mathscr{B}$. On the other hand, they do depend on the interactions being allowed to be "long-range" in the sense that the weighting $g(|X|)$ depends only on the cardinality of $X$ and not on its diameter. In particular, a twobody Ising interaction is in any $\mathscr{B}_{g}$ provided it is summable.

We are concerned with the basic question, "What does a typical phase diagram look like?" According to the Gibbs Phase Rule, an $n$-dimensional subspace of interactions should typically have an $n$-1-dimensional set of two-phase coexistence (i.e. where there are two extremal invariant equilibrium states), an $n-2$ dimensional set of three-phase coexistence, etc. Moreover, one would expect these sets to be manifolds (perhaps with boundary). While these principles may be valid in spaces of short-range interactions, our results show an entirely different picture in $\mathscr{B}_{g}$ : a finite-dimensional subspace which is "typical" in the sense of Baire category will have no points with multiple phases. One may also start out with a given finite-dimensional subspace $S$ of interactions and perturb it, i.e. consider $S+M$, where $M$ is a finite-dimensional subspace. Our result shows that "typically" the only multiple-phase points in $S+M$ are those in $S$ itself.

Actually, the result is even stronger than this: $S$ may be any $\sigma$-compact set, i.e. the union of a countable collection of compact sets. In the quantum case, or the classical discrete-spin case, $S$ may be any Banach space of interactions with a norm

$$
\|\Phi\|_{g, h} \equiv \sum_{0 \in X} g(|X|) h(X)\|\Phi(X)\|
$$

where $h(X) \rightarrow \infty$ as diam $(X) \rightarrow \infty$. (To show this is $\sigma$-compact, note that its unit ball can be approximated by bounded subsets of finite-dimensional subspaces, and therefore is totally bounded.) So we can conclude that any "typical" long-range perturbation destroys the long-range order of all short-range interactions.

There are a number of possible ways to defend the physical Gibbs Phase Rule from the results of this paper. One might say

(1) Physics is not generic, or

(2) Physical interactions are short-ranged.

These objections are related, and both have some merit. Regarding (1), we might remark that the fact that a property of the members of some space is "generic" does not mean we should expect any particular member to have that property, but instead says something about the sort of additional conditions that might ensure that it does not have the property. In this case, it would seem that the only reasonable way to save the Gibbs Phase Rule is to insist on short-ranged interactions.

On the other hand, there are indeed long-range forces in nature, such as the magnetic forces that occur in real ferromagnets. Admittedly, the magnetic dipoledipole interaction does not fit in any of our Banach spaces, but its effects are in a way analogous to those of the perturbing interactions of [1-3] and Theorem 1: while negligible on the atomic scale in comparison to the exchange interaction, on a larger scale it causes the formation of "domains" which interfere with true "ferromagnetic" long-range order. The energy-minimization considerations that govern the formation of magnetic domains have some resemblance to the arguments of these papers. So perhaps our results are not completely devoid of physical significance. 
In Sect. 2 we basically generalize the results of Daniels and van Enter [1-3] on existence of perturbations that destroy long-range order. The main new features are the following:

(1) symmetry is not required, so that we can deal with all interactions and observables;

(2) the conclusions hold for all invariant equilibrium states, not just those that are extremal Gibbs states;

(3) we note that we can perturb not just a single interaction $\Phi$, but a large class of them.

Then in Sect. 3 we combine the existence result with topological arguments to prove the main result (Theorem 2) of this paper. As an application, we prove a result similar to a theorem of Sokal [8].

A closely related result in a more general context of affine continuous functions on a Choquet simplex is Theorem 3.2 of [6]. That theorem includes Theorem 2 of this paper for the space $\mathscr{B}$.

\section{Existence of Order-Destroying Perturbations}

For $\Phi \in \mathscr{B}$ let

$$
J_{N}(\Phi)=\sum_{0 \in X}\|\Phi(X)\||X|^{-1} \min (1, \operatorname{diam}(X) / N) .
$$

Note that the sum converges, and $J_{N}(\Phi) \rightarrow 0$ as $N \rightarrow \infty$ for any $\Phi$. An interaction $\Phi$ may be considered as "short-ranged" if $J_{N}(\Phi) \rightarrow 0$ relatively quickly as $N \rightarrow \infty$. Given any sequence $f_{N}$, we denote by $U_{f}$ the set of interactions $\Phi$ such that $J_{N}(\Phi)<f_{N}$ for all sufficiently large $N$.

Another notion of "short range" is membership in a Banach space $\mathscr{C}_{h}$ of interactions with a norm

$$
N_{h}(\Phi) \equiv \sum_{0 \in X}\|\Phi(X)\||X|^{-1} h(X),
$$

where $h(X)>0$ and $h(X) \rightarrow \infty$ as $\operatorname{diam}(X) \rightarrow \infty$. The following lemma relates membership in this space to decay of $J_{N}(\Phi)$.

Lemma 1. For any $h(X)>0$ with $h(X) \rightarrow \infty$ as $\operatorname{diam}(X) \rightarrow \infty$ there is a sequence $f_{N} \rightarrow 0$ such that $\mathscr{C}_{h} \cong U_{f}$. Conversely, for any sequence $f_{N}>0$ with $f_{N} \rightarrow 0$ as $N \rightarrow \infty$, $U_{f}$ is contained in some Banach space $\mathscr{C}_{h}$.

Proof. Given $h$, let

$$
k_{N}(X)=\frac{\min (1, \operatorname{diam}(X) / N)}{h(X)} .
$$

We have $k_{N}(X) \leqq 1 / h(X) \rightarrow 0$ as $\operatorname{diam}(X) \rightarrow \infty$ for each $N$, while $k_{N}(X) \rightarrow 0$ monotonically as $N \rightarrow \infty$ for each $X$, so it is easy to see that $k_{N}(X) \rightarrow 0$ uniformly in $X$ as $N \rightarrow \infty$. Let $f_{N} \rightarrow 0$ with $\max _{X} k_{N}(X) / f_{N} \rightarrow 0$ as $N \rightarrow \infty$. Now if $\Phi \in \mathscr{C}_{h}$, then

$$
\begin{gathered}
J_{N}(\Phi) \leqq \sum_{0 \in X}\|\Phi(X)\||X|^{-1} h(X) k_{N}(X) \leqq N_{h}(\Phi) \max _{X} k_{N}(X) \\
<f_{N} \text { for } N \text { sufficiently large. }
\end{gathered}
$$


Conversely, given $f_{N}$, let $N_{j}$ be a strictly increasing sequence of integers such that $N_{1}=0$ and

$$
\sum_{j=1}^{\infty} f_{N_{j}}<\infty
$$

and let $h_{0}(X)=j$, where $N_{j} \leqq \operatorname{diam}(X)<N_{j+1}$. If $\Phi \in U_{f}$ then $h_{0}(X) \rightarrow \infty$ as $\operatorname{diam}(X) \rightarrow \infty$ and

$$
\sum_{0 \in X}\|\Phi(X)\| h_{0}(X) /|X|=\sum_{j} \sum_{\substack{0 \in X \\ h_{0}(X)=j}}\|\Phi(X)\| /|X| \leqq \sum_{j=1}^{\infty} J_{N_{j}}(\Phi)<\infty .
$$

Theorem 1. Let $A \in \mathscr{A}_{Y}$ be a local observable, and let $f_{N} \rightarrow 0$ as $N \rightarrow \infty$. Then there is an interaction $\Psi$ of the form

$$
\Psi((x+Y) \cup(y+Y))=J(x-y)\left(\tau_{x} A\right)\left(\tau_{y} A\right), \quad \Psi(X)=0 \text { otherwise }
$$

with all $J(x) \geqq 0$ such that, for every $\Phi \in U_{f}$ and every $t>0$, all the invariant equilibrium states for $\Phi+t \Psi$ agree on $A$. (In the quantum case, if $x+Y$ and $y+Y$ are not disjoint, we replace $\left(\tau_{x} A\right)\left(\tau_{y} A\right)$ by $\left(\left(\tau_{x} A\right)\left(\tau_{y} A\right)+\left(\tau_{y} A\right)\left(\tau_{x} A\right)\right) / 2$ to ensure selfadjointness.) $\Psi$ is in all the spaces $\mathscr{B}_{g}$.

Proof. Let

$$
c_{m}(A)=\left|C_{m}\right|^{-1} \sum_{x \in C_{m}} \tau_{x} A
$$

where $C_{m}$ is a cube of side $m$. We may define an interaction $\Psi_{m}$ of the above form such that $J(x)$ is the sum of the coefficients of terms $\left(\tau_{y} A\right)\left(\tau_{y+x} A\right)$ and $\left(\tau_{y+x} A\right)\left(\tau_{y} A\right)$ in the expansion of $\left(c_{m}(A)\right)^{2}$. We will then have

$$
\varrho\left(A_{\Psi_{m}}\right)=\varrho\left(c_{m}(A)^{2}\right)
$$

for any invariant state $\varrho$. Note that $\left\|\Psi_{m}\right\|_{g} \leqq\|A\|^{2} g(2|Y|)$.

We will take $\Psi=\sum_{j=1}^{\infty} 2^{-j} \Psi_{m_{j}}$, where $m_{j}$ is chosen as follows: Take a sequence $N_{j}$ increasing so rapidly that

$$
\begin{gathered}
N_{j}>N_{j-1}^{4}, \\
2^{j} f_{N_{j}} \rightarrow 0 \text { as } j \rightarrow \infty, \\
2^{2 j} / N_{j} \rightarrow 0 \text { as } j \rightarrow \infty,
\end{gathered}
$$

and let $m_{j}=N_{j}^{2}$. Note that $\|\Psi\|_{g} \leqq\|A\|^{2} g(2|Y|)$.

Now if $\varrho_{1}$ and $\varrho_{2}$ are invariant states with $\left|\varrho_{1}(A)-\varrho_{2}(A)\right| \geqq \varepsilon$, it is easy to show that $\varrho=\left(\varrho_{1}+\varrho_{2}\right) / 2$ satisfies

$$
\varrho\left(c_{m}(A)^{2}\right) \geqq \varrho(A)^{2}+\varepsilon^{2} / 4 .
$$

Of course if $\varrho_{1}$ and $\varrho_{2}$ are equilibrium states for an interaction $\Phi+t \Psi$, the same is true for $\varrho$. To show that $\varrho$ can not be an equilibrium state for this interaction, we will find another invariant state $\varrho$ such that

$$
s(\tilde{\varrho})-\tilde{\varrho}\left(A_{\Phi+t \Psi}\right)>s(\varrho)-\varrho\left(A_{\Phi+t \Psi}\right),
$$

and that will prove the theorem. 
We will obtain $\varrho$ from $\varrho$ by "decoupling" large blocks of the lattice. Given the invariant state $\varrho$ and a positive integer $N$, define $\tilde{\varrho}_{N}$ as follows: cover the lattice by disjoint cubes of side $N$ (which we will call "blocks"). Let $\varrho_{N}$ be the product of the restrictions of $\varrho$ to each block (in probabilistic terminology, we make the blocks independent). Let $\tilde{\varrho}_{N}$ be the average of translations of $\varrho_{N}$ by all elements of a block. Then $\tilde{\varrho}_{N}$ is an invariant state. We wish to prove (2.10) with $\tilde{\varrho}=\tilde{\varrho}_{N_{k}}$ for some $k$. Note that $s\left(\tilde{\varrho}_{N}\right) \geqq s(\varrho)$, so (using linearity) it suffices to prove

$$
\varrho\left(A_{\Phi}\right)+t \varrho\left(A_{\Psi}\right)>\tilde{\varrho}_{N_{k}}\left(A_{\Phi}\right)+t \tilde{\varrho}_{N_{k}}\left(A_{\Psi}\right) \text {. }
$$

The idea is that the difference between $\varrho$ and $\tilde{\varrho}_{N_{k}}$ on $A_{\Phi}$ or on $A_{\Psi_{j}}$ for $j<k$ is a "surface effect," while for $j>k$ we get a "volume effect" which will be dominant if $k$ is sufficiently large. The following estimates will make that precise.

First, there is a constant $K_{1}$ (independent of $\varrho$ and $N$ ) such that if $B \in \mathscr{A}_{X}$, then

$$
\left|\left(\tilde{\varrho}_{N}-\varrho\right)(B)\right| \leqq K_{1}\|B\| \operatorname{diam}(X) / N,
$$

since $\varrho_{N}\left(\tau_{x} B\right)-\varrho(B)=0$ unless $x+X$ intersects more than one block (this is the "surface effect"). This implies that if $\Phi \in U_{f}$

$$
\left|\left(\tilde{\varrho}_{N}-\varrho\right)\left(A_{\Phi}\right)\right| \leqq K_{1} J_{N}(\Phi) \leqq K_{1} f_{N}
$$

for $N$ sufficiently large. Moreover, there is a constant $K_{2}$ (depending only on $A$ and $X$ ) such that

$$
\left|\left(\varrho_{N}-\varrho\right)\left(A_{\Psi_{m}}\right)\right|=\left|\left(\varrho_{N}-\varrho\right)\left(c_{m}(A)^{2}\right)\right|<K_{2} m / N .
$$

Since $N_{k}>m_{j}^{2}$ for $j<k$, this implies

$$
\left|\left(\tilde{\varrho}_{N_{k}}-\varrho\right)\left(\sum_{j<k} 2^{-j} A_{\Psi_{m_{j}}}\right)\right|<K_{2} N_{k}^{-1 / 2} .
$$

On the other hand, there is a constant $K_{3}$ (depending only on $A$ and $X$ ) such that

$$
\left|\varrho_{N}\left(c_{m}(A)^{2}\right)-\varrho(A)^{2}\right| \leqq K_{3}\left(\frac{1}{N}+\left(\frac{N}{m}\right)^{d}\right) .
$$

[The $1 / N$ comes from those terms $\varrho_{N}\left(\tau_{x}\left(\tau_{y} A \cdot \tau_{z} A\right)\right)$ in the expansion where $x+y+X$ or $x+z+X$ intersects more than one block, the $(N / m)^{d}$ from those where $x+y+X$ and $x+z+X$ are contained in the same block.] Thus for $j \geqq k$, using (2.9) and $m_{j} \geqq N_{k}^{2}$, we get

$$
\left(\tilde{\varrho}_{N_{k}}-\varrho\right)\left(A_{\Psi_{m_{j}}}\right)<-\frac{\varepsilon^{2}}{4}+\frac{2 K_{3}}{N_{k}}<-\frac{\varepsilon^{2}}{8}
$$

if $k$ is sufficiently large. From (2.13), (2.15), and (2.17), then,

$$
\left(\tilde{\varrho}_{N_{k}}-\varrho\right)\left(A_{\Phi}+t A_{\Psi}\right)<K_{1} f_{N_{k}}+t\left(K_{2} N_{k}^{-1 / 2}-2^{-k-2} \varepsilon^{2}\right),
$$

and by (2.7) and (2.8) this is negative for $k$ sufficiently large.

\section{Genericity}

The following lemma shows that the members of any $\sigma$-compact set of interactions may be considered "short-ranged" in the sense of Sect. 2. Recall that a $\sigma$-compact set is defined as a countable union of compact sets. 
Lemma 2. Every $\sigma$-compact subset of $\mathscr{B}_{g}$ is in some $U_{f}$.

Proof. Let $S=\bigcup_{n=1}^{\infty} S_{n}$ with $S_{n}$ compact. $J_{N}$ is continuous on $\mathscr{B}_{g}$ and decreases pointwise to 0 as $N \rightarrow \infty$, so by Dini's Theorem the convergence is uniform on the compact sets $S_{n}$. Take an increasing sequence $N_{m}$ so that $J_{N}(\Phi)<1 / m$ if $\Phi \in \bigcup_{n=1}^{m} S_{n}$
and $N \geqq N_{m}$, and define $f_{N}=1 / m$ if $N_{m} \leqq N<N_{m+1} . \quad \square$

Now we come to the main result of this paper. Much of the proof is practically identical to the first part of the proof of Theorem 3.2 of [6], but is included here for the sake of completeness.

Theorem 2. Let $S$ be any $\sigma$-compact subset of $\mathscr{B}_{g}$, and for each positive integer $n$ let $G_{n}$ be the set of $n$-tuples $\Psi=\left(\Psi_{1}, \ldots, \Psi_{n}\right) \in\left(\mathscr{B}_{g}\right)^{n}$ with the following property: for every $\Phi \in S$ and every $c \in \mathbb{R}^{n} \backslash\{0\}$, the interaction $\Phi+c \cdot \Psi=\Phi+\sum c_{i} \Psi_{i}$ has a unique invariant equilibrium state. Then $G_{n}$ is a dense $G_{\delta}$ set in $\left(\mathscr{B}_{g}\right)^{n}$.

Proof. For each local observable $A$ (i.e. $A \in \mathscr{A}_{Y}$ for some finite $Y$ ), let $G_{n}^{A}$ be defined in the same way as $G_{n}$, except that instead of a unique invariant equilibrium state we require that all invariant equilibrium states agree on $A$. By separability and the fact that local observables are dense in $\mathscr{A}, G_{n}$ is a countable intersection of sets $G_{n}^{A}$, so it suffices to prove each of these is a dense $G_{\delta}$.

Let $C$ be the set of interactions in $\mathscr{B}_{g}$ which have invariant equilibrium states that differ on $A$, and $C_{k}$ be the set of interactions in $\mathscr{B}_{g}$ which have invariant equilibrium states that differ on $A$ by at least $1 / k$. Using compactness of the set of invariant states, it is easy to see that $C_{k}$ is closed. By writing $S=\cup S_{i}$ and $R^{n} \backslash\{0\}$ $=\cup K_{j}$, where the $S_{i}$ and $K_{j}$ are compact, we can express $G_{n}^{A}$ as a countable intersection of sets

$$
H_{i, j, k}=\left\{g \in\left(\mathscr{B}_{g}\right)^{n}: C_{k} \cap\left(S_{i}+K_{j} \cdot g\right)=\emptyset\right\} .
$$

If $g \in H_{i, j, k}$, then $\operatorname{dist}\left(S_{i}+K_{j} \cdot g, C_{k}\right)>0$. It follows that $H_{i, j, k}$ is open, and hence that $G_{n}^{A}$ is a $G_{\delta}$.

In order to prove that $G_{n}^{A}$ is dense, we will prove that $G_{1}^{A}$ is dense and then use the following induction argument: If $\left(g_{1}, \ldots, g_{n-1}\right) \in G_{n-1}^{A}$, let $S^{\prime}=S$ $+\operatorname{span}\left\{g_{1}, \ldots, g_{n-1}\right\}$, which is again $\sigma$-compact. If $g_{n} \in G_{1}^{A}$ (based on $S^{\prime}$ instead of $S)$, then it is easy to see that $\left(g_{1}, \ldots, g_{n-1}, g_{n}\right) \in G_{n}^{A}$ (based on $\left.S\right)$. So if $G_{n-1}^{A}$ is dense in $\left(\mathscr{B}_{g}\right)^{n-1}$ and all sets of the form $G_{1}^{A}$ are dense in $\mathscr{B}_{g}$, it follows that $G_{n}^{A}$ is dense in $\left(\mathscr{B}_{g}\right)^{n}$.

In order to prove that $G_{1}^{A}$ is dense, write $G_{1}^{A}=G_{+}^{A} \cap G_{-}^{A}$, where $G_{+}^{A}$ (respectively $G_{-}^{A}$ ) is defined in the same way as $G_{1}^{A}$, but with the restriction $c>0$ (respectively $c<0)$. The same argument as above shows that these are $G_{\delta}$ sets, and it suffices to prove $G_{+}^{A}$ is dense. (Then $G_{-}^{A}=-G_{+}^{A}$ is dense as well.) Since increasing $S$ decreases $G_{+}^{A}$, we may assume that $S$ is a dense linear subspace (noting that $\mathscr{B}_{g}$ is separable and the linear span of a $\sigma$-compact set is $\sigma$-compact). Then if $\Psi \in G_{+}^{A}$, it follows that $G_{+}^{A}$ contains the dense set $\Psi+S$. Thus it suffices to show that $G_{+}^{A}$ is nonempty. But by Lemma $2, S$ is contained in some $U_{f}$, and then Theorem 1 provides an interaction $\Psi \in G_{+}^{A}$ as required. 
Among the consequences of the existence of order-destroying perturbations are results similar to those of Sokal [8]. The result we present below, being based on strict convexity of the pressure, requires stronger conditions on the function $g$ than does Sokal's.

Theorem 3. Suppose $g(n) \geqq 1$ (classical case) or $g(n) \geqq e^{a n}$ for some $a>0$ (quantum case). Let Vbe a finite-dimensional subspace of $\mathscr{B}_{g}$, and let $\Phi_{0} \in \mathscr{B}_{g}$. Then for any $\Psi$ in a dense $G_{\delta}$ set in $\mathscr{B}_{g}$ and any invariant equilibrium state $\varrho$ for $\Phi_{0}$, there is a continuous curve $\Phi_{t} \in \Phi_{0}+t \Psi+V$ for $-\infty<t<\infty$ passing through $\Phi_{0}$, such that for all $t \neq 0$ the unique invariant equilibrium state $\varrho_{t}$ for $\Phi_{t}$ agrees with $\varrho$ on all the observables $A_{v}$ for $v \in V$.

Proof. Since $\Phi_{0}+V$ is $\sigma$-compact, Theorem 2 provides the dense $G_{\delta}$ set of $\Psi$ such that all interactions in $\Phi_{0}+t \Psi+V$ for $t \neq 0$ have unique invariant equilibrium states.

By first applying a projection, we may assume that there are no nonzero elements of $V$ that are physically equivalent to 0 . This implies that the "pressure" $P$ is strictly convex on $\Phi_{0}+t \Psi+V$ for any $t$ [8, Sect. III.4]. Thus $\Phi_{0}$ is the unique minimum of $P-\alpha$ on $\Phi_{0}+V$, where $\alpha(\Phi)=-\varrho\left(A_{\Phi}\right)$. Now (since $V$ is finitedimensional) $P-\alpha \rightarrow \infty$ as $\|v\| \rightarrow \infty$ on $\Phi_{0}+V$. Since $P$ is uniformly Lipschitz, this is also true on $\Phi_{0}+t \Psi+V$ for any $t$, and that implies that $P-\alpha$ attains a minimum on this space. Let that minimum be at $\Phi_{t}$. Then the unique invariant equilibrium state at $\Phi_{t}$ agrees with $\varrho$ on $A_{v}$ for $v \in V$. The continuity of $\Phi_{t}$ follows easily from the strict convexity of $P$ on $\Phi_{0}+t \Psi+V$ : given $t$ and $\varepsilon>0$, note that $\inf \left\{(P-\alpha)\left(\Phi_{t}+v\right):\|v\| \geqq \varepsilon\right\}>(P-\alpha)\left(\Phi_{t}\right)$. Then for $|s|$ sufficiently small, $(P-\alpha)\left(\Phi_{t}+s \Psi+v\right)>(P-\alpha)\left(\Phi_{t}+s \Psi\right)$ for $\|v\| \geqq \varepsilon$. This implies that $\left\|\Phi_{t+s}-\left(\Phi_{t}+s \Psi\right)\right\|<\varepsilon$.

In particular, if $A_{v}$ for $v \in V$ separate the invariant equilibrium states at $\Phi_{0}$ then $\varrho_{t} \rightarrow \varrho$ in the weak-* topology as $t \rightarrow 0$. Such a $V$ will exist if the set of invariant equilibrium states at $\Phi_{0}$ is finite-dimensional. If, on the other hand, that set is infinite-dimensional, we may take an increasing sequence of spaces $V_{n}$ whose union is dense in $\mathscr{B}_{g}$, choose $\Phi_{n}$ on the curve given above for $V_{n}$ so that $\Phi_{n} \rightarrow \Phi_{0}$ as $n \rightarrow \infty$, and obtain a result similar to that of Sokal:

Corollary. For any invariant equilibrium state $\varrho$ for an interaction $\Phi_{0} \in \mathscr{B}_{g}$, there is a sequence $\Phi_{n} \rightarrow \Phi_{0}$ in $\mathscr{B}_{g}$ for which the invariant equilibrium states are unique, and tend to $\varrho$ in the weak-* topology.

Acknowledgement. I would like to thank Joel Lebowitz for his hospitality at Rutgers University, where much of this work was done.

\section{References}

1. Daniels, H.A.M., van Enter, A.C.D.: Differentiability properties of the pressure in lattice systems. Commun. Math. Phys. 71, 65-76 (1980)

2. van Enter, A.C.D.: A note on the stability of phase diagrams in lattice systems. Commun. Math. Phys. 79, 25-32 (1981)

3. van Enter, A.C.D.: Stability properties of phase diagrams in lattice systems (thesis), Rijksuniversiteit te Groningen (1981) 
4. Israel, R.B.: Convexity in the theory of lattice gases. Princeton Series in Physics. Princeton, NJ: Princeton University Press 1979

5. Israel, R.B.: Existence of phase transitions for long-range interactions. Commun. Math. Phys. 43, 59-68 (1975)

6. Israel, R.B., Phelps, R.R.: Some convexity questions arising in statistical mechanics. Math. Scand. 54, 133-156 (1984)

7. Ruelle, D.: A heuristic theory of phase transitions. Commun. Math. Phys. 53, 195-208 (1977)

8. Sokal, A.: More surprises in the general theory of lattice systems. Commun. Math. Phys. 86, 327-336 (1982)

Communicated by J. Fröhlich

Received January 20, 1986; in revised form April 7, 1986 